\title{
Intracellular adhesion molecule-1 modulates $\beta$-chemokines and directly costimulates $T$ cells in vivo
}

\author{
Jong J. Kim, ${ }^{1}$ Anthony Tsai, ${ }^{1}$ Liesl K. Nottingham, ${ }^{2}$ Lake Morrison, ${ }^{1}$ Devin M. Cunning, ${ }^{2}$ \\ Jim Oh, ${ }^{1}$ Daniel J. Lee, ${ }^{1}$ Kesen Dang, ${ }^{1}$ Tzvete Dentchev, ${ }^{1}$ Ara A. Chalian, ${ }^{2}$ \\ Michael G. Agadjanyan, ${ }^{1}$ and David B. Weiner ${ }^{1,3}$
}

${ }^{1}$ Department of Pathology and Laboratory Medicine, and

${ }^{2}$ Department of Otolaryngology/Head and Neck Surgery, University of Pennsylvania, Philadelphia, Pennsylvania 19104, USA

${ }^{3}$ The Wistar Institute, Philadelphia, Pennsylvania 19104, USA

Address correspondence to: David B. Weiner, Department of Pathology and Laboratory Medicine, University of Pennsylvania, 505 Stellar-Chance, 422 Curie Boulevard, Philadelphia, Pennsylvania 19104, USA. Phone: (215) 349-8365; Fax: (215) 573-9436; E-mail: dbweiner@mail.med.upenn.edu

Received for publication December 10, 1998, and accepted in revised form February 2, 1999.

\begin{abstract}
The potential roles of adhesion molecules in the expansion of $\mathrm{T}$ cell-mediated immune responses in the periphery were examined using DNA immunogen constructs as model antigens. We coimmunized cDNA expression cassettes encoding the adhesion molecules intracellular adhesion molecule-1 (ICAM-1), lymphocyte function associated-3 (LFA-3), and vascular cell adhesion molecule-1 (VCAM-1) along with DNA immunogens, and we analyzed the resulting antigen-specific immune responses. We observed that antigenspecific T-cell responses can be enhanced by the coexpression of DNA immunogen and adhesion molecules ICAM-1 and LFA-3. Coexpression of ICAM-1 or LFA-3 molecules along with DNA immunogens resulted in a significant enhancement of T-helper cell proliferative responses. In addition, coimmunization with pCICAM-1 (and more moderately with PCLFA-3) resulted in a dramatic enhancement of CD8-restricted cytotoxic T-lymphocyte responses. Although VCAM- 1 and ICAM- 1 are similar in size, VCAM- 1 coimmunization did not have any measurable effect on cell-mediated responses. These results suggest that ICAM1 and LFA-3 provide direct T-cell costimulation. These observations are further supported by the finding that coinjection with ICAM- 1 dramatically enhanced the level of interferon- $\gamma($ IFN- $\gamma$ ) and $\beta$-chemokines macrophage inflammatory protein- $1 \alpha$ (MIP- $1 \alpha)$, MIP- $1 \beta$, and regulated on activation normal T-cell expression and secreted (RANTES) produced by stimulated T cells. Through comparative studies, we observed that ICAM-1/LFA-1 T-cell costimulatory pathways are independent of CD86/CD28 pathways and that they may synergistically expand T-cell responses in vivo.
\end{abstract}

J. Clin. Invest. 103:869-877 (1999).

\section{Introduction}

The immune response is the result of complex molecular interplay of both soluble signaling molecules and immune cell-expressed surface molecules. Among these molecules, chemokines are highly bioactive inflammatory molecules that play a major role in a variety of immune and inflammatory responses, acting primarily as chemoattractants and activators of various leukocytes (1-3). To carry out these functions, chemokines regulate a variety of molecules. Among the important molecules regulated by chemokine expression are adhesion molecules. As the name suggests, adhesion molecules are thought to play critical roles in both cell migration and cell-cell contact during immune activation and expansion. During immune or inflammatory response, lymphocytes leave the circulation and migrate to the site of antigen exposure. Adhesion molecules on lymphocytes and endothelial cells mediate direct cellular contact, directing the migration and trafficking of the leukocytes. In addition, adhesion molecules play an important role in the binding of $\mathrm{T}$ lymphocytes to antigen-presenting cells (APCs).

T-cell activation is a complex process that has several requirements. One requirement is for the T-cell receptor (TCR) complex to identify and bind to an activated APC based on the specificity of the individual TCR for a specific peptide-MHC molecules displayed on the surface of an APC. In addition, secondary (costimulatory) signals provided through the ligation of costimulatory molecules with their receptors on $T$ cells are required for efficient T-cell activation. Among different costimulatory molecules, CD80 and CD86 have been observed to provide potent immune signals $(4,5)$. As surface glycoproteins and members of the immunoglobulin superfamily, the CD80 and CD86 molecules are expressed only on professional APCs and interact with their receptors CD28 and CTLA-4 present on T cells (4-7).

Although adhesion molecules participate in immune expansion, their functions as costimulators of this process rather than secondary players remain to be clarified (8-10). Among the most important adhesion molecules on T-cell surface in its interaction with APCs are lymphocyte function associated-1 (LFA-1) and CD2 (11). These molecules bind to intracellular adhesion molecules (ICAMs; ICAM-1, -2, and -3) and LFA-3, respectively, on the APCs. It is thought that the adhesive interaction between these molecules is crucial for T cells to sample large numbers of specific peptides presented on MHC on the surface of the APC (11). ICAM-1 (CD54) is a surface molecule expressed 

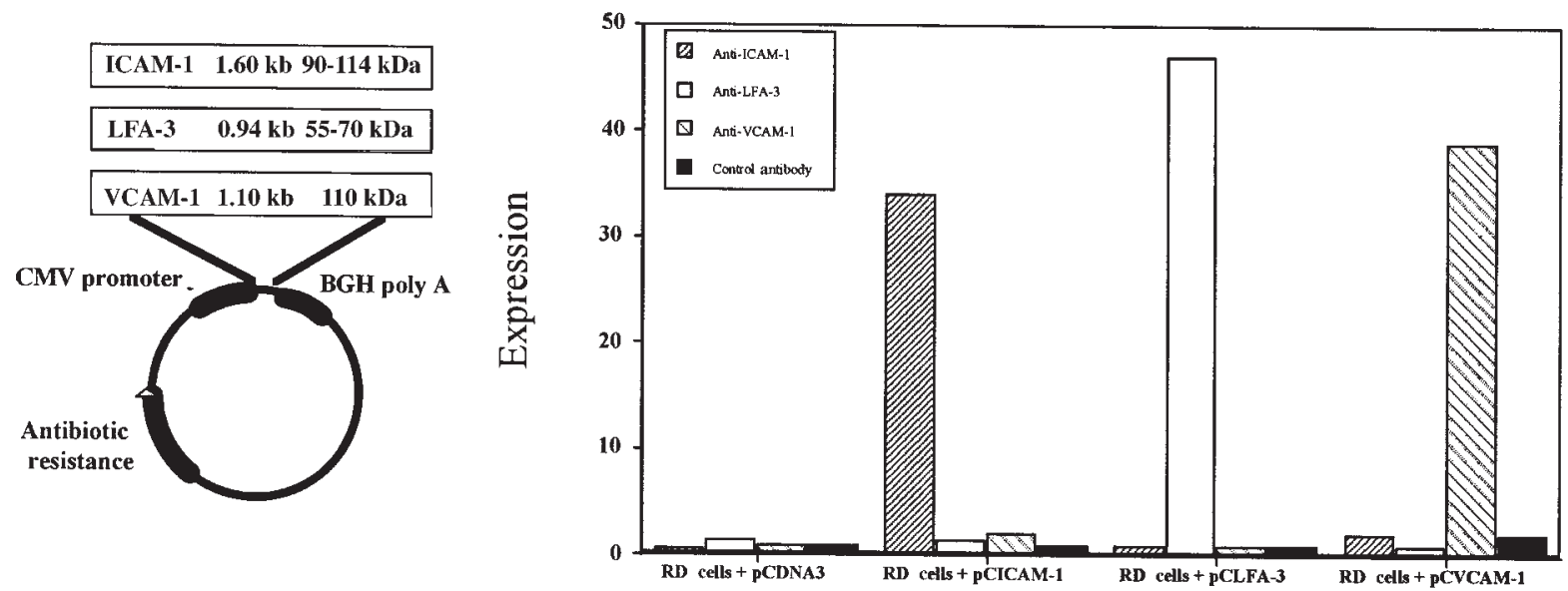

Figure 1

Expression of pCICAM-1 and pCLFA-3 on human rhabdomyosarcoma $(R D)$ cells in vitro. RD cells were transfected with pCDNA3 (control), pCICAM1 , or PCLFA-3. Three days after transfection, the cells were removed from the plates and were analyzed by FACS ${ }^{\circledR}$ analysis using either control antibodies, $\alpha$-intracellular adhesion molecule-1 (ICAM-1), or $\alpha$-lymphocyte function associated-3 (LFA-3) antibodies to detect expression of the transfected gene product.

on endothelial cells, macrophages, and dendritic cells. Its ligand, LFA-1, is expressed on activated T cells, thus trapping $T$ cells at the site of inflammation and possibly playing a facilitating role in antigen presentation and T-cell costimulation. A similar set of molecules with perhaps overlapping functions are the LFA-3/CD2 cell-surface antigens. LFA-3 is expressed on the surface of macrophages and dendritic cells and could complement T-cell costimulation through linking the T cell to the APC. To date, however, no direct effect on immunogenicity has been ascribed to the molecules, and as such, their role in T-cell expansion is largely inferential. Another important adhesion molecule is vascular cell adhesion molecule-1 (VCAM-1; CD106). VCAM-1 is expressed on activated endothelial cells and is thought to play a role in lymphocyte trafficking through binding the very late antigen-4 (VLA-4) ligand.

We recently have reported (12) that chemokines can regulate antigen-specific immune expansion of DNAencoded antigen. Because chemokines strongly drive adhesion molecule expression, this suggests that certain adhesion molecules could play a role in this specific immune expansion. Accordingly, we examine here the immunomodulatory effects of three specific adhesion molecules that are closely regulated by chemokines. We used DNA vaccine technology to explore the role of ICAM-1, LFA-3, and VCAM-1 in immune activation in vivo. Specifically, we cloned genes for ICAM-1, LFA-3, and VCAM- 1 individually into expression vectors under control of a cytomegalovirus (CMV) promoter. These constructs were then coimmunized along with DNA immunogens encoding for HIV-1 envelope or gag/pol antigens. We analyzed the immunological effects of the coinjection with these adhesion molecule cassettes on the level of antigen-specific immune responses.

We observed that antigen-specific T-cell responses can be enhanced by the coexpression of DNA immunogen and adhesion molecules ICAM- 1 and LFA-3. ICAM- 1 and LFA-3, however, appeared to play no role in expression of antigen-specific humoral responses. Rather, they appeared to specifically affect T-cell responses. LFA-3 enhanced
$\mathrm{CD} 4^{+} \mathrm{T}$-cell responses and exhibited more minor effect on CD8 ${ }^{+}$T-cell function. More importantly, ICAM-1 coadministration dramatically increased both $\mathrm{CD}^{+}$and $\mathrm{CD} 8^{+}$ T-cell responses. ICAM-1 coexpression also dramatically enhanced antigen-specific $\beta$-chemokine production, suggesting an important role for ligation of LFA- 1 in peripheral T-cell expansion (12). The activation phenotype of these molecules appeared to be distinct from the prototypic CD80/CD86 costimulatory molecules. These results support that the peripheral network of cytokine, chemokine, and adhesion molecules coordinately regulate effector T-cell responses at the site of effector function.

\section{Methods}

DNA plasmids. DNA vaccine constructs expressing HIV-1 envelope protein (pCEnv) and gag/pol protein (pCGag/pol) were prepared as described previously (13). The genes for ICAM-1, LFA-3, and VCAM-1 were cloned into the PCDNA3 expression vector (Invitrogen Corp., San Diego, California, USA) as described previously $(13,14)$. Clean plasmid DNA was produced as described previously (14).

Reagents and cell lines. Human rhabdomyosarcoma (RD) and mouse mastocytoma P815 cell lines were obtained from American Type Culture Collection (Rockville, Maryland, USA). Recombinant vaccinia expressing HIV-1 envelope (vMN462), $\mathrm{gag} / \mathrm{pol}(\mathrm{vVK} 1)$, and $\beta$-galactosidase (vSC8) were obtained from the National Institutes of Health AIDS Research and Reference Reagent Program. Recombinant gp120 or p24 protein were obtained from ImmunoDiagnostics Inc. (Bedford, Massachusetts, USA).

Expression of adhesion molecule expression constructs. Expression of ICAM-1, LFA-3, and VCAM-1 constructs were analyzed by transfecting them into RD cells as described previously (15). Cells were harvested $72 \mathrm{~h}$ after transfection and tested for expression using FACS $^{\circledR}$ analysis with FITC-conjugated monoclonal antibodies for ICAM-1, LFA-3, VCAM-1 (PharMingen, San Diego, California, USA) (15).

DNA inoculation of mice. The quadriceps muscles of 6- to 8week-old female BALB/c mice (Harlan Sprague Dawley Inc., Indianapolis, Indiana, USA) were injected with $50 \mu \mathrm{g}$ of each DNA construct of interest formulated in PBS and $0.25 \%$ bupivacaine-HCl (Sigma Chemical Co., St. Louis, Missouri, USA). Coadministration of various gene expression cassettes involved 
$a$

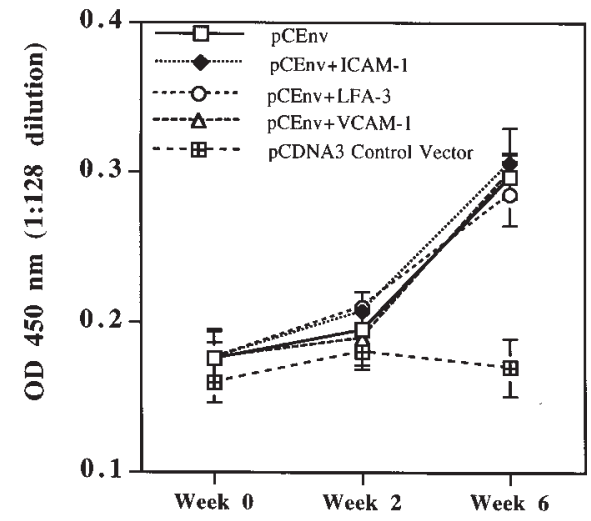

Time

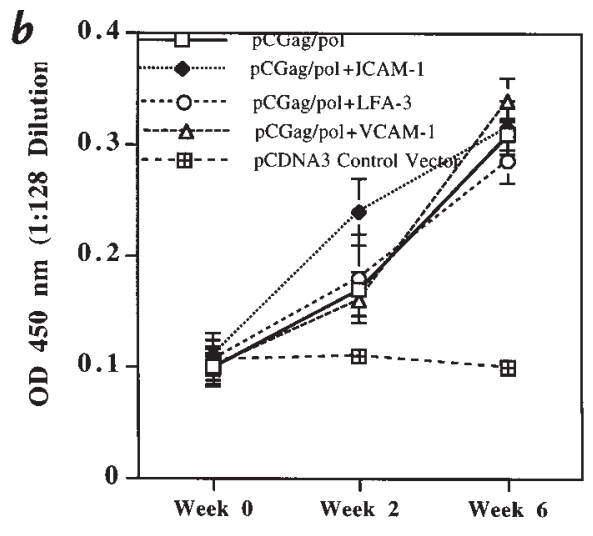

Time

Figure 2

HIV-1 envelope and gag/pol-specific antibody response after coimmunization with pCICAM-1 or pCLFA-3. The mouse serum samples were tested at 1:128 dilution for antigen-specific antibody response using the ELISA against (a) HIV-1 gp120 protein and (b) HIV-1 p24 protein.

mixing the chosen plasmids before injection. The control mice were immunized with $50 \mu \mathrm{g}$ of pCDNA3 vector. Each set of studies was performed three times, and a representative set of results is presented. Mice received two DNA immunization (50 $\mu \mathrm{g}$ each) separated by 2 weeks. At 1 week after the boost injection, the mice were sacrificed, the spleens were harvested, and the lymphocytes were isolated and tested for cellular (T-helper cell [Th] or cytotoxic T lymphocyte [CTL]) responses.

ELISA. Fifty microliters of $\mathrm{p} 24$ or gp 120 protein diluted in 0.1 $\mathrm{M}$ carbonate-bicarbonate buffer ( $\mathrm{pH} 9.5$ ) to $2 \mu \mathrm{g} / \mathrm{ml}$ concentration was adsorbed onto microtiter wells overnight at $4^{\circ} \mathrm{C}$ as described previously (13). The plates were washed with PBS $/ 0.05 \%$ Tween-20 and blocked with 3\% BSA in PBS with $0.05 \%$ Tween- 20 for $1 \mathrm{~h}$ at $37^{\circ} \mathrm{C}$. Mouse antisera was diluted with $0.05 \%$ Tween-20, incubated for $1 \mathrm{~h}$ at $37^{\circ} \mathrm{C}$, and then incubated with horseradish peroxidase-conjugated goat anti-mouse IgG (Sigma Chemical Co.). The plates were washed and developed with $3^{\prime} 3^{\prime} 5^{\prime} 5^{\prime}$ TMB (Sigma Chemical Co.) buffer solution. The plates were read on a Dynatech MR5000 plate reader (Dynatech Corp., Burlington, Massachusetts, USA) with the OD at $450 \mathrm{~nm}$.

$\mathrm{Th}$ proliferation assay. Lymphocytes were harvested from spleens and prepared as the effector cells by removing the erythrocytes and by washing several times with fresh media as described (13). The isolated cell suspensions were resuspended to a concentration of $5 \times 10^{6}$ cells $/ \mathrm{ml}$. A $100-\mathrm{ml}$ aliquot containing $5 \times 10^{5}$ cells was immediately added to each well of a 96-well microtiter flat-bottom plate. Recombinant p24 or gp120 protein at the final concentration of $5 \mu \mathrm{g} / \mathrm{ml}$ and 1 $\mu \mathrm{g} / \mathrm{ml}$ was added to wells in triplicate. The cells were incubated at $37^{\circ} \mathrm{C}$ in $5 \% \mathrm{CO}_{2}$ for 3 days. Tritiated thymidine (1 $\mu \mathrm{Ci}$ ) was added to each well, and the cells were incubated for $12-18 \mathrm{~h}$ at $37^{\circ} \mathrm{C}$. The plate was harvested, and the amount of incorporated tritiated thymidine was measured in a Beta Plate reader (Wallac, Turku, Finland). Stimulation index (SI) was determined from the following formula: $\mathrm{SI}=($ experimental count / spontaneous count).

Spontaneous-count wells include $10 \%$ FCS, which serves as an irrelevant protein control. In addition, pCEnv or control immunized animals routinely have SI of 1 against $\operatorname{Pr} 55$ protein. Similarly, pCGag/pol or control routinely have SI of 1 against gp120 protein. To ensure that cells were healthy, PHA or concanavalin A (Con A) (Sigma Chemical Co.) was used as a polyclonal stimulator positive control. The PHA or Con A control samples had a SI of 20-40.

CTL assay. A 5-h ${ }^{51} \mathrm{Cr}$ release CTL assay was performed using vaccinia-infected targets (13). The assay was performed with in vitro effector stimulation, whereby the effectors were stimulated with relevant vaccinia-infected cells (vMN462 for envelope and $\mathrm{vVK} 1$ for $\mathrm{gag} / \mathrm{pol}$ ) that were fixed with $0.1 \%$ glutaraldehyde for 5 days in CTL culture media at $5 \times 10^{6}$ cells $/ \mathrm{ml}$. The effectors were
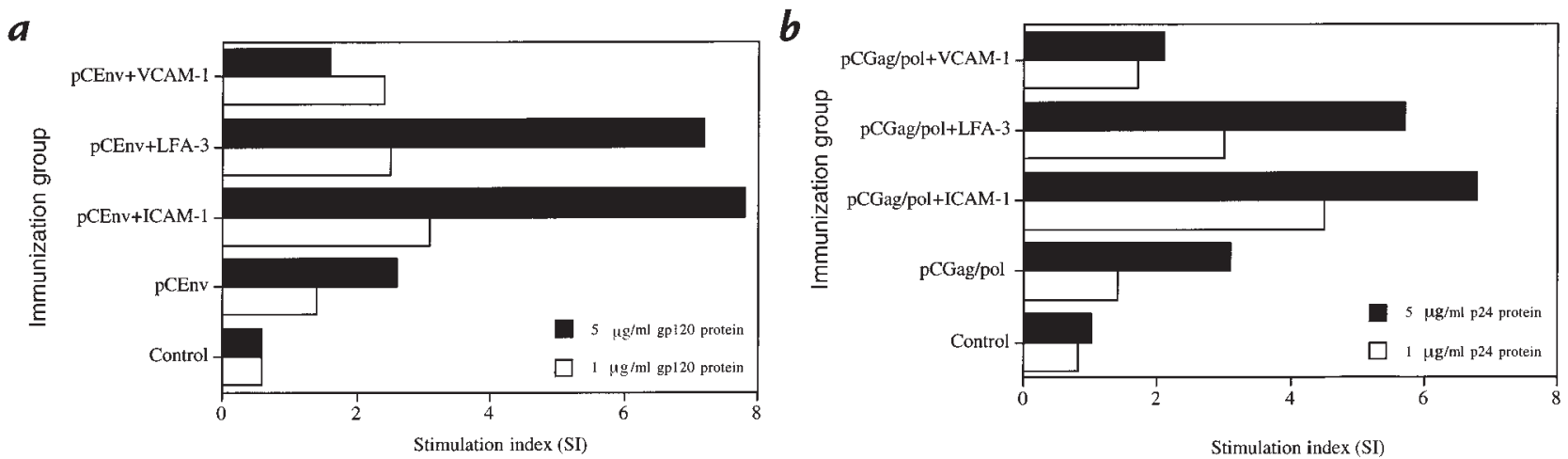

Figure 3

T-helper cell proliferation responses after coinjection with adhesion molecule genes. Mouse lymphocytes were isolated as described in Methods and tested against $(\boldsymbol{a})$ recombinant gp120 protein ( 5 and $1 \mu \mathrm{g} / \mathrm{ml}$ final concentrations) or against $(\boldsymbol{b})$ recombinant p24 protein $(5$ and $1 \mu \mathrm{g} / \mathrm{ml}$ final concentrations). Background counts per minute from the control vector group were 200-400. 
$a$

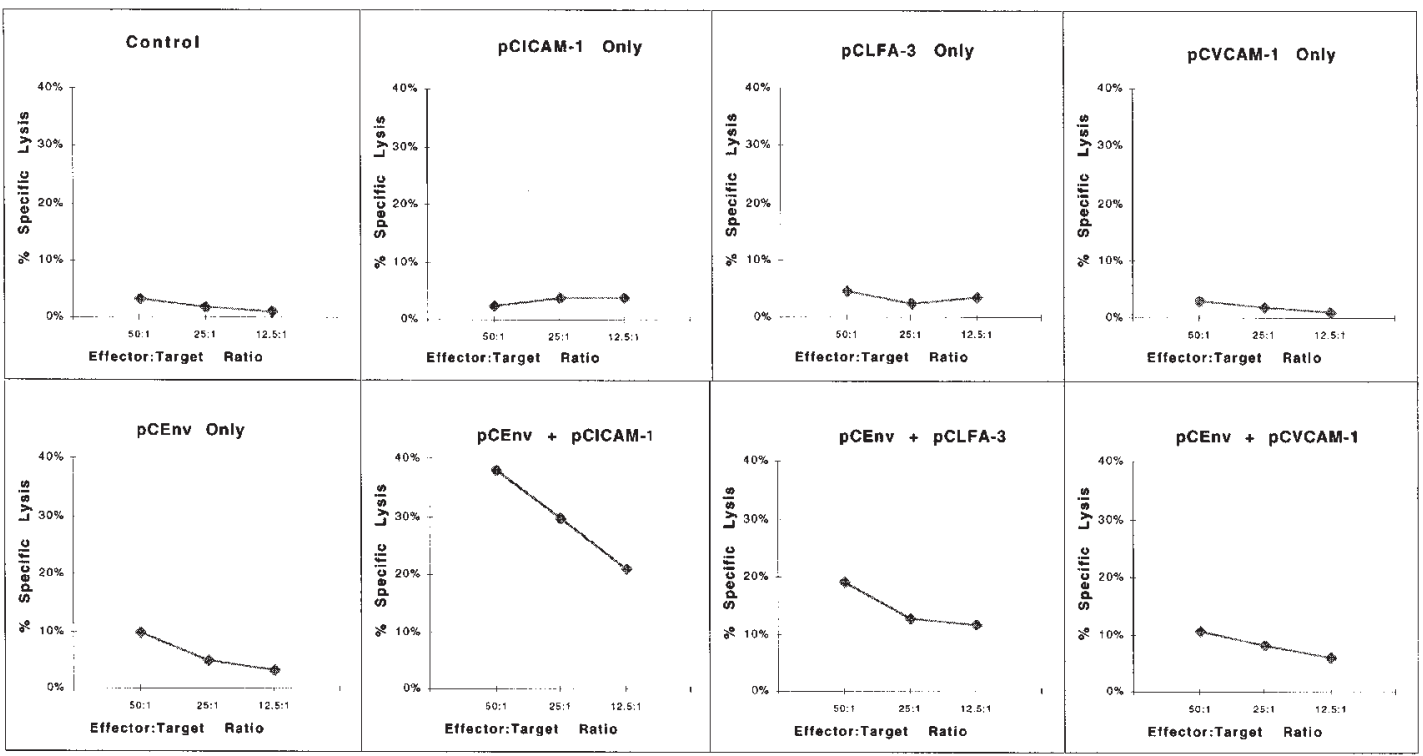

$\boldsymbol{b}$

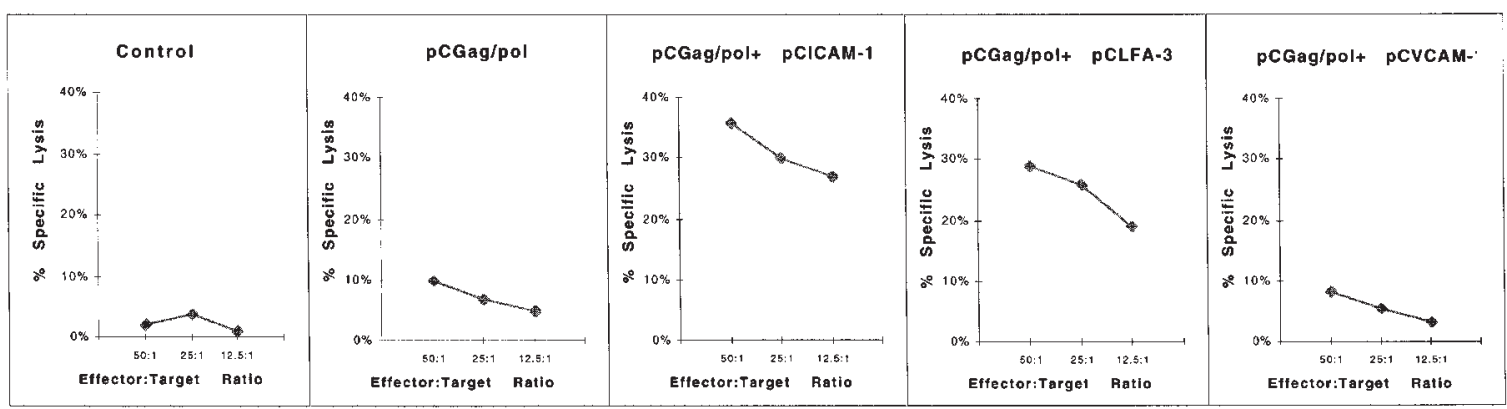

Figure 4

Antigen-specific CTL responses following coinjection with adhesion molecule genes. Mouse lymphocytes were isolated as described in Methods and tested for CTL response using ( $\boldsymbol{a}$ ) target cells infected with envelope-specific (vMN462) and nonspecific (vSC8) vaccinia; and (b) target cells infected with gag-specific (vVK1) and nonspecific (vSC8) vaccinia. CTL, cytotoxic T lymphocyte.

a

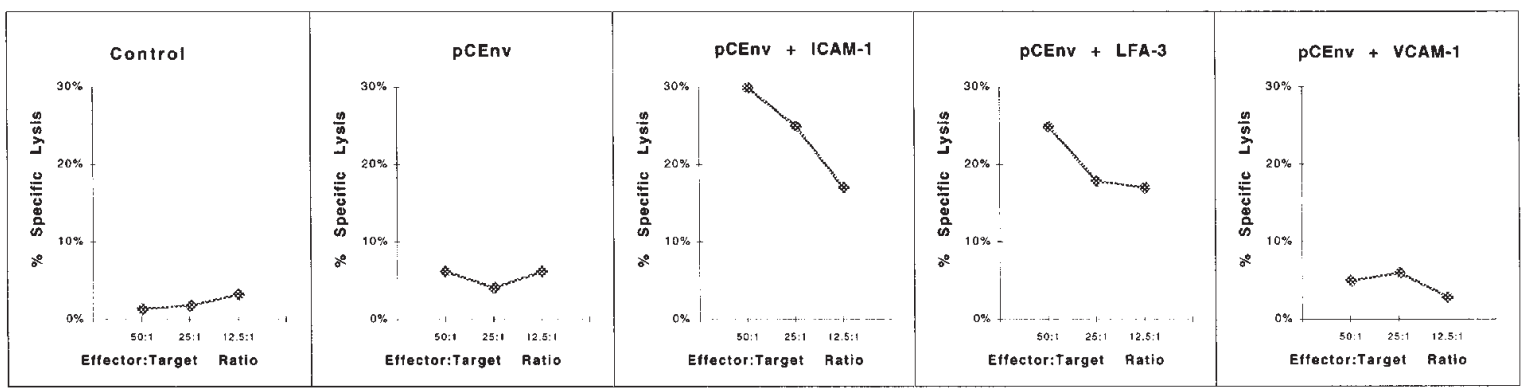

$\boldsymbol{b}$

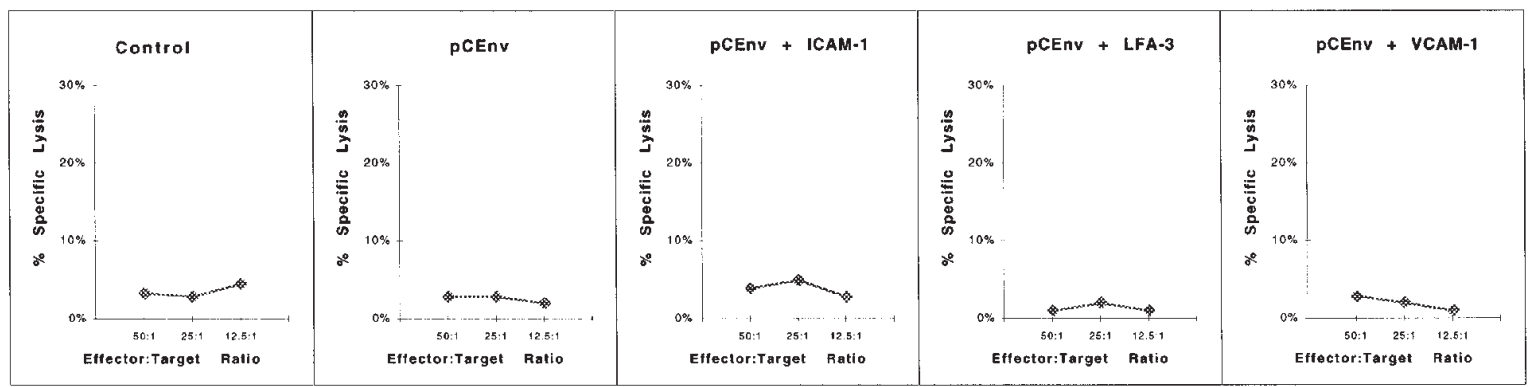

Figure 5

Determination of CD8 $8^{+}$-dependent CTLs. A CTL assay was performed with the removal of CD8 ${ }^{+} T$ cells by complement lysis. Effector cells were prepared as described with the presence of CD8 ${ }^{+} T$ cells $(\boldsymbol{a})$ and the absence of CD8 $8^{+} T$ cells $(\boldsymbol{b})$ using vaccinia-infected target cells. 
stimulated nonspecifically for 2 days with CTL culture media consisting of RPMI-1640 (GIBCO BRL, Grand Island, New York, USA), 10\% FCS (GIBCO BRL), and 10\% RAT-T-STIM without Con A (Becton Dickinson Labware, Bedford, Massachusetts, USA). Vaccinia-infected targets were prepared by infecting $3 \times 10^{6}$ P815 cells at the multiplicity of infection of 10-20 for 5-12 h at $37^{\circ} \mathrm{C}$. A standard chromium-release assay was performed in which the target cells were labeled with $100 \mu \mathrm{Ci} / \mathrm{ml} \mathrm{Na}_{2}{ }^{51} \mathrm{CrO}_{4}$ for 60-120 min and used to incubate with the stimulated effector splenocytes for $4-6 \mathrm{~h}$ at $37^{\circ} \mathrm{C}$. CTL lysis was determined at effector/target $(\mathrm{E} / \mathrm{T})$ ratios ranging from 50:1 to 12.5:1. Supernatants were harvested and counted. Percent specific lysis was determined from the formula:

$$
100 \times\left(\frac{\text { experimental release }- \text { spontaneous release }}{\text { maximum release }- \text { spontaneous release }}\right)
$$

Maximum release was determined by lysis of target cells in medium containing $1 \%$ Triton X-100. An assay was not considered valid if the value for the spontaneous release counts was in excess of $20 \%$ of the maximum release.

Complement lysis of $C D 8^{+} T$ cells. $\mathrm{CD}^{+} \mathrm{T}$ cells were removed from the splenocytes by a treatment with $\alpha$-CD 8 monoclonal antibody (PharMingen) followed by incubation with rabbit complement (Sigma Chemical Co.) for $45 \mathrm{~min}$ at $37^{\circ} \mathrm{C}$ as described (13).

Cytokine and chemokine expression analysis. Supernatants from effectors stimulated for CTL assay were collected at day 6 and tested for expression using ELISA kits for IFN- $\gamma$; IL-4; and MIP-1 $\alpha$, MIP-1 $\beta$, and RANTES (BioSource International, Camarillo, California, USA; R\&D Systems, Minneapolis, Minnesota, USA; Intergen, Purchase, New York, USA, respectively).

\section{Results}

ICAM-1, LFA-3, and VCAM-1 can be expressed by transfected cells. The genes for ICAM-1 (pCICAM-1), LFA-3 (pCLFA3 ), and VCAM-1 (pCVCAM-1) were individually cloned into the pCDNA3 expression vector (Fig. 1). To test whether pCICAM-1, pCLFA-3, and pCVCAM-1 constructs could express their relevant proteins, we transfected them in vitro into the human $\mathrm{RD}$ cells. Using $\mathrm{FACS}^{\circledR}$ analysis, we observed that transfection of PCICAM-1, pCLFA-3, and pCVCAM-1 expression cassettes resulted in specific expression of ICAM-1, LFA-3, and VCAM-1, respectively (Fig. 1). We also observed that coimmunization of two DNA expression cassettes intramuscularly resulted in coexpression of both encoded proteins in same muscle cells in vivo (data not shown).

Coexpression of adbesion molecules does not affect antigenspecific humoral immune responses. We next investigated the effects the coexpression of adhesion molecules have on the induction of antigen-specific immune responses. For all experiments, $50 \mu \mathrm{g}$ of each DNA expression constructs was injected into BALB/c mice intramuscularly at weeks 0 and 2 . The first immune parameter examined was the antigen-specific humoral response. Antisera from immunized mice were collected at weeks 0,2 , and 6 and were analyzed for specific antibody responses against HIV-1 gp120 protein by enzymelinked immunosorbent assay. As shown in Fig. 2a, coexpression of ICAM-1, LFA-3, or VCAM-1 appeared to have a minimal effect on the specific antibody binding profile induced by pCEnv immunization. A similar result was seen with the groups coimmunized with pCGag/pol (Fig. 2b).

\section{a}

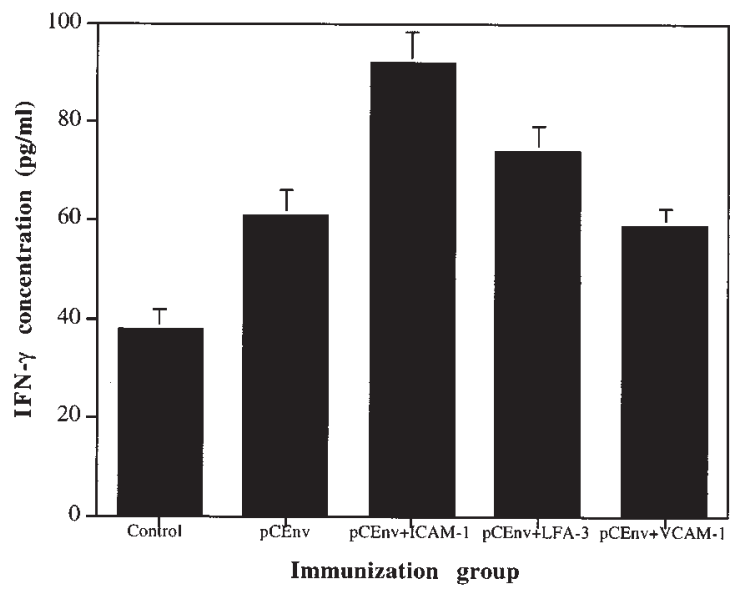

$b$

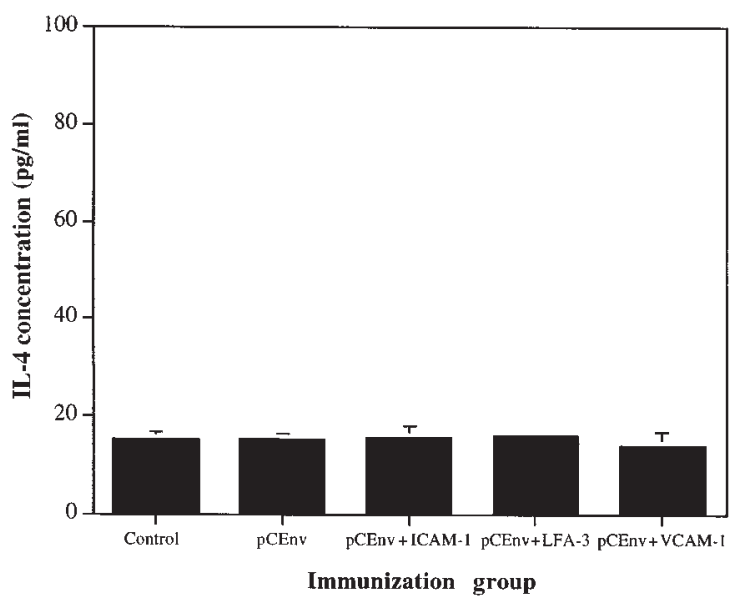

Figure 6

Expression of cytokines by stimulated effector T cells. Supernatants from effectors stimulated for CTL assay were collected at day 6 and tested for cytokine profile using ELISA kits for interferon- $\gamma($ IFN- $\gamma)(a)$ and interleukin-4 (IL-4) (b).

Coexpression of ICAM-1 or LFA-3 enhances antigen-specific Th proliferative response. The effect of adhesion molecule coexpression on the magnitude of cellular immune responses was also investigated. Induction of $\mathrm{CD}^{+} \mathrm{Th}$ proliferative response is important because Th play a critical role in inducing both a humoral immune response via B cells and CTL response via $\mathrm{CD}^{+} \mathrm{T}$ cells. Figure $3 a$ shows the Th proliferative responses for the mice immunized with pCGag/pol and those mice coimmunized with pCICAM1, pCLFA-3, or pCVCAM-1. Recombinant gp120 HIV-1 envelope protein $(5 \mu \mathrm{g} / \mathrm{ml}$ and $1 \mu \mathrm{g} / \mathrm{ml})$ was plated in each well for specific stimulation of T-cell proliferation. We also analyzed these groups for nonspecific stimulation of $\mathrm{T}$ cells using irrelevant proteins and observed that nonspecific antigen did not induce T-cell proliferative responses in vitro (data not shown). As shown in Fig. $3 a$, a background level of proliferation was observed in the control group immunized with a control vector, and a moderate level of proliferation was observed in the group immunized with pCEnv alone as we have reported previously (15). In contrast, the groups coimmunized with either PCICAM-1 or pCLFA-3 had significantly higher levels of proliferative responses. On the other hand, the group coimmunized 


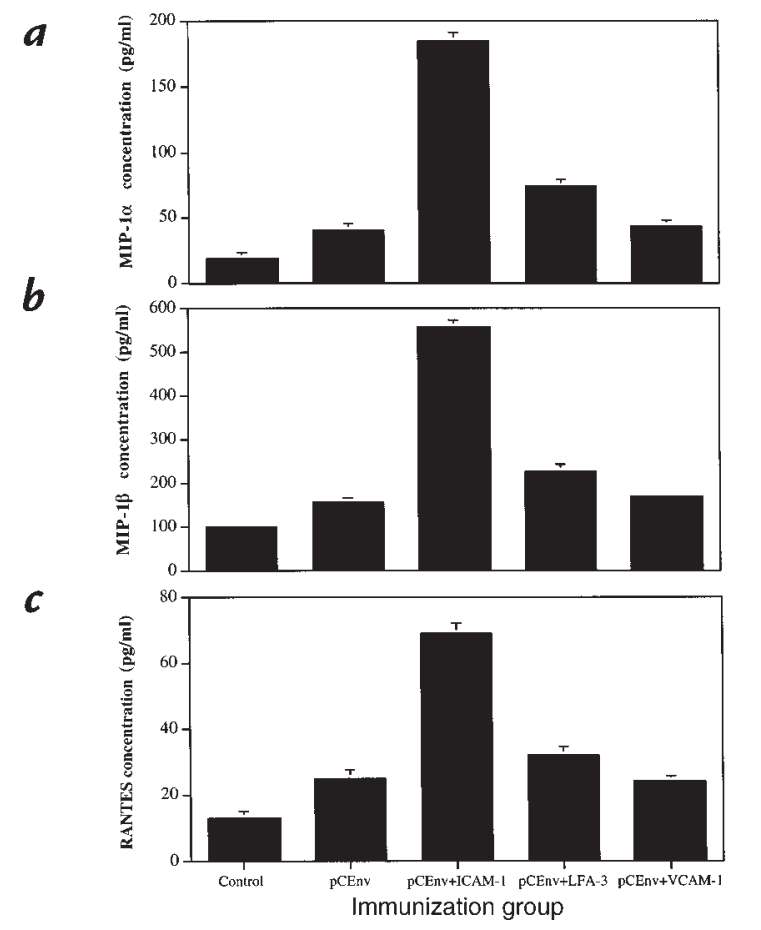

Figure 7

Expression of $\beta$-chemokines by stimulated effector T cells. Supernatants from effectors stimulated for CTL assay were collected at day 6 and tested for $\beta$-chemokine profile using ELISA kits for macrophage inflammatory protein-macrophage inflammatory protein-1 $\alpha(M I P-1 \alpha)(a)$, macrophage inflammatory protein-1 $\beta(M I P-1 \beta)(b)$, and regulated on activation normal T-cell expression and secreted (RANTES) (c).

with VCAM-1 genes did not show any enhancement of antigen-specific Th response. A similar result was seen with the groups coimmunized with pCGag/pol (Fig. 3b). In repeat experiments using either immunogen, codelivery of pCICAM-1 or PCLFA-3 resulted in a three- to fourfold increase in antigen-specific proliferative responses.

Coexpression of ICAM-1 or LFA-3 enhances antigen-specific CTL responses. To investigate further the enhancement of cellular immunity, we performed CTL assays using splenocytes of mice coimmunized with pCEnv and pCGag/pol. The assay was performed with in vitro stimulation of effector splenocytes before measuring chromium release from specific and nonspecific vaccinia-infected or peptide-treated targets (15). To calculate specific lysis of targets, the percent lysis of irrelevant targets was subtracted from the percent lysis of specific targets. A background level of specific killing was observed from the control animals with pCDNA3, pCICAM-1, pCLFA-3, or pCVCAM-1 immunizations, whereas the animals immunized with pCEnv showed a low level of CTL response (Fig. 4a). On the other hand, coimmunization with pCEnv + pCICAM-1 resulted in a dramatic increase in CTL activity. Greater than $40 \%$ specific killing of HIV-1 envelope vaccinia (vMN462)-infected targets was observed after coimmunization with pCEnv + pCICAM-1 at a 50:1 $\mathrm{E} / \mathrm{T}$ ratio. The CTL activity titered out to $20 \%$ specific lysis at a $12.5: 1 \mathrm{E} / \mathrm{T}$ ratio. In contrast, coimmunization with pCEnv + pCLFA -3 resulted in a more moderate increase in CTL activity. Similar CTL results were observed after coimmunizations with $\mathrm{pCGag} / \mathrm{pol}+\mathrm{pCICAM}-1$ and pCGag/pol + pCLFA-3 (Fig. 4b).

To determine whether the increases in CTL response via coexpression of PCICAM- 1 and PCLFA-3 were restricted to $\mathrm{CD}^{+} \mathrm{T}$ cells, CTL assays were performed by measuring CTL activity with and without the removal of $\mathrm{CD}^{+} \mathrm{T}$ cells from the effector cell population by complement lysis (13). As shown in Fig. 5, the removal of $\mathrm{CD}^{+} \mathrm{T}$ cells resulted in the suppression of antigen-specific CTL enhancement observed after coinjections with PCICAM- 1 and PCLFA-3. These results indicate that the enhancement of cytolytic activity was antigen-specific and $\mathrm{CD} 8^{+} \mathrm{T}$ cell-dependent.

Coexpression of ICAM-1 or LFA-3 increases production of IFN- $\gamma$ by stimulated T cells. Analysis of cytokine production by stimulated CTLs in the immunized animals support the CTL results just described. Cytokines play a key role in directing and targeting immune cells during the development of the immune response. For instance, IFN- $\gamma$ is intricately involved in the regulation of $\mathrm{T}$ cell-mediated cytotoxic immune responses (16), whereas IL-4 plays a dominant role in B cell-mediated immune responses (17). We analyzed supernatant from the effector cells stimulated in vitro for CTL assay and tested them for the release of cytokines IFN- $\gamma$ and IL-4. We found that coinjection with pCICAM-1 increased the level of IFN- $\gamma$ significantly (Fig. 6). Coimmunization with PCLFA-3 resulted in a more moderate increase in IFN- $\gamma$ production. On the other hand, the level of IL-4 released from all groups was similar.

Coexpression of ICAM-1 dramatically increases production of $\beta$ chemokines by stimulated $T$ cells. Recently, we reported that $\mathrm{CD}^{+}$effector $\mathrm{T}$ cells expand antigen-specific responses in vivo through the production of specific chemokines at the peripheral site of infection (12). Therefore, we analyzed the production of $\beta$-chemokines by stimulated CTLs. We analyzed supernatant from the effector cells stimulated in vitro for CTL assay and tested them for the release of $\beta$ chemokines MIP- $1 \alpha$, MIP- $1 \beta$, and RANTES. As we had previously observed, we found that DNA immunization with pCEnv induced significantly greater levels of expression of MIP- $1 \alpha$, MIP- $1 \beta$, and RANTES than those of control vector (Fig. 7). Moreover, we observed that coinjection with pCEnv + pCLFA-3 increased the level of $\beta$-chemokine production compared with that of the pCEnv immunized group. Even more significantly, coimmunization with pCEnv + pCICAM-1 resulted in a dramatic enhancement (two- to fourfold) of MIP- $1 \alpha$, MIP-1 $\beta$, and RANTES production over that of the pCEnv immunized group. In contrast, coadministration of PCVCAM-1 did not enhance the level of chemokine expression. These results support that ICAM-1 and LFA-3 provide direct T-cell costimulation.

Coexpression of ICAM-1 and CD86 synergistically enhances antigen-specific CTL responses. The B7 (CD80 and CD86) pathway is considered to be a major costimulatory pathway for the delivery of critical second signals to prime and expand T-cell responses. These molecules have been examined in the context of DNA vaccines as modulatory agent. In this context, it appears that CD86 molecules play a prominent role in the antigen-specific induction of $\mathrm{CD} 8^{+}$cytotoxic $\mathrm{T}$ lymphocytes when delivered as vaccine adjuvants $(13,18,19)$. Coadministration of CD86 cDNA along with DNA immunogens dramatically increased antigen-specific CD8 ${ }^{+} \mathrm{CTL}$ response (13). The effects of 
ICAM- 1 and LFA-3 could be dependent on B7-CD28 signals, or they could represent an alternative synergistic pathway for driving CTL induction in vivo. We further investigated whether ICAM-1 and LFA-3 molecules when coexpressed with CD86 molecules could synergistically enhance the level of CTL induction. As shown in Fig. 8, we observed that coexpression of ICAM-1 and CD86 molecules could synergistically enhance antigen-specific CTL response. On the other hand, coexpression of LFA-3 and CD86 molecules did not improve the level of CTL response. These results indicate that ICAM-1/LFA-1 pathways provide T-cell costimulatory signals independent of CD86/CD28 pathways, and they may work synergistically to expand T-cell responses in vivo.

The level of IFN- $\gamma$ and $\beta$-chemokines MIP- $1 \alpha$, MIP- $1 \beta$, and RANTES production by stimulated CTLs further supports these results, indicating that ICAM-1/LFA-1 signals work independent of CD86/CD28 signals and work concordantly to expand T-cell responses. When we analyzed supernatant from the effector $\mathrm{T}$ cells using the methods already described here, we observed that coadministration of LFA-3 and CD86 genes resulted in a dramatically higher level of IFN- $\gamma$, MIP- $1 \alpha$, MIP- $1 \beta$, and RANTES (Fig. 9). These results further imply the synergistic nature of ICAM-1 and CD86 in T-cell activation.

\section{Discussion}

During immune or inflammatory response, lymphocytes traffic to the site of antigen exposure. Adhesion molecules on lymphocytes and endothelial cells play an important role in providing direct cellular contact and directing the migration of the leukocytes. In addition, adhesion molecules play an important role in the binding of $\mathrm{T}$ lymphocytes to APCs. ICAM-1 (CD54) is a 90- to $114-\mathrm{kDa}$ molecule that is expressed on endothelial cells, macrophages, and dendritic cells and binds to LFA-1 and Mac-1 (20). Almost all leukocytes, including T lymphocytes, express LFA-1, whereas Mac-1 expression is more restricted to monocytes, macrophages, and granulocytes. LFA-3 (CD58) is a 55- to 70-kDa surface molecule expressed by various cell types, including the APCs (11). VCAM-1 is a $110-\mathrm{kDa}$ surface molecule that is expressed on activated endothelial cells and smooth muscle cells (21). VCAM-1 recognizes and binds to VLA-4, which is constitutively expressed on most mononuclear leukocytes, including the eosinophils, lymphocytes, monocytes, and basophils, but is absent on neutrophils (22). VCAM-1/VLA-4 interaction plays an important role in leukocyte migration and diapedesis.

In this study, we used a DNA immunogen model to investigate the roles of these cell-surface adhesion molecules in providing stimulatory signals required for $\mathrm{T}$-cell activation and expansion. In a two-signal T-cell activation model, the primary activation signal is mediated by the ligation of antigenic peptide-MHC molecules to T-cell receptor. The secondary costimulatory signal is provided through the ligation of CD80/CD86 costimulatory molecules with their receptors (CD28/CTLA-4) present on T cells (6). Although this two-signal model is conceptually straightforward and well supported by experimental results, the costimulatory signals provided during $\mathrm{T}$-cell activation process may not be restricted only to the B7
(CD80/CD86) molecules. Additional cell-surface molecules, such as the adhesion molecules on the APCs, may also have an important function in providing costimulation, and their roles in providing direct signals to $\mathrm{CD}^{+}$ and $\mathrm{CD}^{+} \mathrm{T}$ cells are under investigation $(8-10,23-25)$.

Adhesion molecules are important in leukocyte trafficking, inflammatory cell recruitment, and immune surveillance. Recently, a role for adhesion molecules in $\mathrm{T}$-cell activation has been suggested. We investigated the

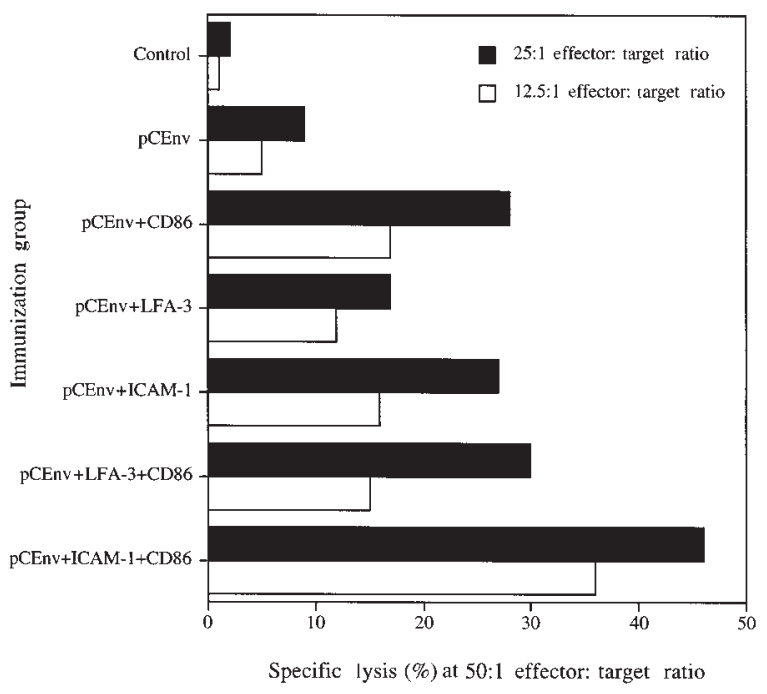

Figure 8

Antigen-specific CTL responses after coinjection with genes for adhesion molecules and CD86 costimulatory molecules. Mouse lymphocytes were isolated as described in Methods and tested for CTL response using target cells infected with envelope-specific (vMN462) and nonspecific (vSC8) vaccinia.

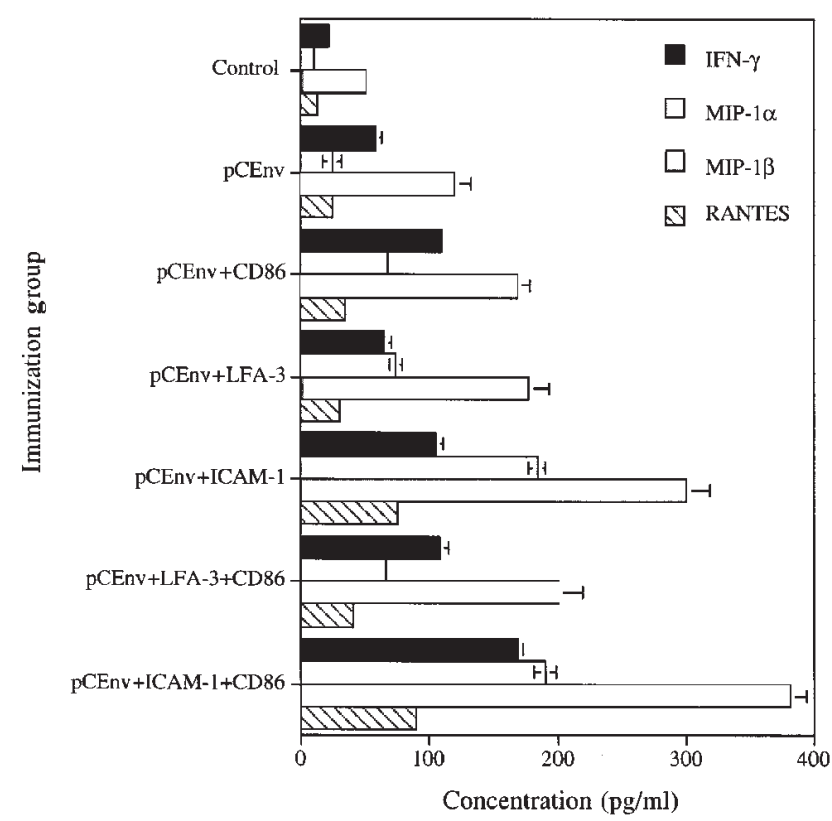

\section{Figure 9}

Expression of IFN- $\gamma$ and $\beta$-chemokines MIP- $1 \alpha$, MIP- $1 \beta$, and RANTES by stimulated effector $T$ cells. Supernatants from effectors stimulated for CTL assay were collected at day 6 and tested using ELISA kits. 
role using a subset of adhesion molecules that all bind to ligands on $\mathrm{T}$ cells. We chose three related molecules: ICAM-1 (CD54), LFA-3 (CD58), and VCAM-1 (CD106). Using DNA expression cassettes encoding for ICAM-1, LFA-3, and VCAM-1 along with our DNA immunogens, we sought to identify the specific effects of coexpressing adhesion molecules along with antigens. We observed that antigen-specific T-cell (both $\mathrm{CD}^{+}$and $\mathrm{CD}^{+} \mathrm{T}$ cells) responses can be enhanced by the coexpression of DNA immunogen and adhesion molecules ICAM-1 and LFA3. Coexpression of ICAM-1 or LFA-3 molecules along with DNA immunogens resulted in a significant enhancement of Th proliferative responses. In addition, coimmunization with pCICAM-1 (and more moderately with pCLFA-3) resulted in a dramatic enhancement of CD8-restricted CTL responses. These observations were further supported by the finding that coinjection with LFA-3 increased the level of IFN- $\gamma$ as well as $\beta$ chemokines MIP- $1 \alpha$, MIP- $1 \beta$, and RANTES production by stimulated $\mathrm{CD}^{+} \mathrm{T}$ cells. More impressively, coimmunization with ICAM-1 resulted in a more dramatic enhancement of IFN- $\gamma$ and $\beta$-chemokines. It is also important to note that increased cellular contact or juxtaposition of cells alone was not enough to enhance antigen-specific $\mathrm{T}$ cell-mediated responses. Although ICAM-1 and VCAM-1 have similar molecular sizes, the coinjection with VCAM-1 did not have any measurable effect on T-cell responses. On the other hand, ICAM-1 coexpression dramatically enhanced the level of both $\mathrm{CD}^{+}$and $\mathrm{CD}^{+} \mathrm{T}$-cell responses. These results imply that the T-cell stimulatory effects are not inherent to their adhesion properties or the size of the molecules. It is interesting that both CTL-driving adhesion molecules that enhanced CTLs (ICAM-1 and LFA-3) are expressed on a variety of APCs. In fact, it may be important that the best CTL-inducing adhesion molecule, ICAM-1, is expressed on dendritic cells.

We also compared the enhanced induction of CTLs with that enhanced with CD86 expression. We observed that combining the expression of CD86 molecules with ICAM-1, but not LFA-3, molecules could enhance antigen-specific CTL responses. These results were further supported by significantly enhanced production of IFN- $\gamma$ as well as $\beta$-chemokines MIP- $1 \alpha$, MIP- $1 \beta$, and RANTES, which play important role in immune activation in the periphery. Although the elucidation of the biologic significance of these molecules requires further studies, a recent study (12) found a relationship between chemokines MIP-1 $\beta$ and RANTES and CTL response. Although additional studies could provide more insight into the costimulatory role of these molecules, these results indicate that ICAM-1 molecules can provide Tcell costimulatory signals through an independent pathway to CD86, and they may work synergistically to amplify the total level of costimulatory signals provided to $T$ cells. Overall, these results support that adhesion molecules ICAM-1 and LFA-3 can provide important costimulatory signals, indicating that the simple twosignal model of T-cell activation, although conceptually useful, may be incomplete, and a newer model with multiple sources of costimulation should be further considered and studied. These results also indicate that further studies aimed at using the T-cell costimulatory function of other cell-surface molecules are warranted.

One important issue with regard to these studies is exactly where these molecules are functioning to enhance the cellular immune response. Recent studies (26-28) have reported that injection of plasmid DNA can, with low efficiency, transfect resident APCs including macrophages and dendritic cells. These results are further supported by studies using bone marrow chimeras that illustrate the requirement for bone marrow-derived cells to prime DNA immune responses $(29,30)$. It is consistent with the literature that some costimulation observed in our study can occur through transfection and enhanced T-cell priming by resident professional APCs.

Along with previous reports, these results support the role for ICAM-1 and LFA-3 in T-cell costimulation. It appears that LFA-3 has particular effects on class II responses, whereas in general, ICAM-1 was a dramatically strong driver of CTL induction and $\mathrm{CD}^{+}$effector function, as demonstrated by enhanced production of $\beta$ chemokines. These results also support a concordant hypothesis for the recruitment and expansion of T-cell effectors in the periphery. We recently reported that in addition to their chemoattractant functions, chemokines regulate modulation and expansion of antigen-specific immune responses at the peripheral site (12). We observed that $\mathrm{CD}^{+} \mathrm{T}$-effector cells control chemokine expression levels while they primed immune responses. Thus, in chemotaxis, chemokines regulate the movement of lymphocytes through a concentration gradient. Moreover, commensurate redistribution of adhesion molecule expression provide direct cell-cell contact in directing the lymphocytes to the periphery. In addition, expression of adhesion molecules is modulated by various inflammatory cytokines and chemokines. For instance, IFN- $\gamma$ and TNF- $\alpha$ have been shown to upregulate ICAM- 1 expression on endothelial and muscle cells $(31,32)$.

$\mathrm{CD}^{+}$effector $\mathrm{T}$ cells elaborate chemokines that would recruit more APCs and T cells to the site of inflammation. These T cells would be stimulated by $\beta$-chemokine production to enhance expression of adhesion molecules, which could serve to drive IFN- $\gamma$ production and allow Tcell costimulation. Once at the site of inflammation, these effector CTLs can be further regulated through the expression of specific chemokines and adhesion molecules that would expand the level of effector function. These results further support that end-stage effector $T$ cells in the expansion phase of an antigen-specific immune response could direct their destiny through coordinated expression and release of these molecules.

\section{Acknowledgments}

This work was supported in part by grants from the National Institutes of Health to D.B. Weiner and M.G. Agadjanyan. We thank Richard Ciccarelli from Wyeth-Lederle Vaccines \& Pediatrics for thoughtful discussion and providing material for this study.

\footnotetext{
1. Baggiolini, M., Beatrice, D., and Moser, B. 1997. Human chemokines: an update. Annu. Rev. Immunol. 15:675-705.

2. Schall, T.J., and Bacon, K.B. 1994. Chemokines, leukocyte trafficking, and inflammation. Curr. Opin. Immunol. 6:865-873.

3. Strieter, R.M., et al. 1996. "The good, the bad, and the ugly." The role of
} 
chemokines in models of human disease. I. Immunol. 156:3583-3586.

4. Lanier, L.L., et al. 1995. CD80 (B7) and CD86 (B70) provide similar costimulatory signals for $\mathrm{T}$ cell proliferation, cytokine production, and generation of CTL. J. Immunol. 154:97-105.

5. Linsley, P.S., Clark, E.A., and Ledbetter, J.A. 1990. The T cell antigen, CD28, mediates adhesion with B cells by interacting with activation antigen, B7/BB-1. Proc. Natl. Acad. Sci. USA. 87:5031-5035.

6. Schwartz, R.H. 1992. Costimulation of T lymphocytes: the role of CD29, CTLA-4, and B7/BB1 in interleukin-2 production and immunotherapy. Cell. 71:1065-1068.

7. June, C., Bluestone, J.A., Nadler, L.M., and Thompson, C.B. 1994. The B7 and CD28 receptor families. Immunol. Today. 15:321-333.

8. Lamphear, J. G., Stevens, K.R., and Rich, R.R. 1998. Intercellular adhesion molecule- 1 and leukocyte function-associated antigen-3 provide costimulation for superantigen-induced $\mathrm{T}$ lymphocyte proliferation in the absence of a specific presenting molecule. J. Immunol. 160:615-623.

9. Zuckerman, L.A., Pullen, L., and Miller, J. 1998. Functional consequences of costimulation by ICAM-1 on IL-2 gene expression and T cell activation. J. Immunol. 160:3259-3268.

10. Gollob, J.A., et al. 1996. Molecular interaction between CD58 and CD2 counter-receptors mediates the ability of monocytes to augment $\mathrm{T}$ cell activation by IL-12. J. Immunol. 157:1886-1893.

11. Springer, T.A., Dustin, M.L., Kishimoto, T.K., and Marlin, S.D. 1987. The lymphocyte function-associated LFA-1, CD-2, and LFA-3 molecules: cell adhesion receptors of the immune system. Annu. Rev. Immunol. 5:223-252.

12. Kim, J.J., et al. 1998. CD8 positive T cells controls antigen-specific immune responses through the expression of chemokines. J. Clin. Invest. 102:1112-1124.

13. Kim, J.J., et al. 1997. Engineering of in vivo immune responses to DNA immunization via co-delivery of costimulatory molecule genes. Nat. Biotechnol. 15:641-645.

14. Kim, J.J., et al. 1998. Modulation of amplitude and direction of in vivo immune responses by co-administration of cytokine gene expression cassettes with DNA immunogens. Eur. J. Immunol. 28:1089-1103.

15. Kim, J.J., et al. 1997. In vivo engineering of a cellular immune response by co-administration of IL-12 expression vector with a DNA immunogen. J. Immunol. 158:816-826.

16. Mogensen, S.C., and Virelizier, J.L. 1987. The interferon-macrophage alliance [review]. Interferon. 8:55-84.

17. Seder, R.A., and Paul, W.E. 1994. Acquisition of lymphokine-producing phenotype by CD4+ T cells. Annu. Rev. Immunol. 12:635-673

18. Iwasaki, A., Stiernholm, B.J., Chan, A.K., Berstein, N.L., and Barber, B.H. 1997. Enhanced CTL responses mediated by plasmid DNA immunogens encoding costimulatory molecules and cytokines. J. Immunol.
158:4591-4601.

19. Tsuji, T., et al. 1997. Immunomodulatory effects of a plasmid expressing B7-2 on human immunodeficiency virus-1-specific cell-mediated immunity induced by a plasmid encoding the viral antigen. Eur. J. Immunol. 27:782-787

20. Dustin, M.L., Rothlein, R., Bhan, A.K., Donarello, C.A., and Springer, T.A. 1986. A natural adherence molecule (ICAM-1): induction by IL-1 and interferon-gamma, tissue distribution, biochemistry, and function. J. Immunol. 137:245-254.

21. Osborn, L., et al. 1989. Direct expression cloning of vascular cell adhesion molecule 1 , a cytokine-induced endothelial protein that binds to lymphocytes. Cell. 59:1203-1211.

22. Elices, M.J., et al. 1990. VCAM-1 on activated endothelium interacts with the leukocyte integrin VLA-4 at a site distinct from the VLA4/fibronectin binding site. Cell. 60:577-584.

23. Parra, E., Wingren, A.G., Hedlund, G., Kalland, T., and Dohlsten, M. 1997. The role of B7-1 and LFA-3 in costimulation of CD8+ T cells. J. Immunol. 158:637-642.

24. Croft, M., and Dubey, C. 1997. Accessory molecule and costimulation requirements for CD4 T cell response. Crit. Rev. Immunol. 17:89-118.

25. Le Guiner, S., et al. 1998. LFA-3 co-stimulates cytokine secretion by cytotoxic T lymphocytes by providing a TCR-independent activation signal. Eur. J. Immunol. 28:1322-1331.

26. Chattergoon, M.A., Robinson, T.A., Boyer, J.D., and Weiner, D.B. 1998. Specific immune induction following DNA-based immunization through in vivo transfection and activation of macrophages. J. Immunol. 160:5707-5718.

27. Casares, S., Inaba, K., Brumeanu, T.D., Steinman, R.M., and Bona, C.A. 1997. Antigen presentation by dendritic cells after immunization with DNA encoding a major histocompatibility complex class II-restricted viral epitope. J. Exp. Med. 186:1481-1486.

28. Condon C., Watkins, S.C., Celluzzi, C.M., Thompson, K., and Falo, L.D. 1996. DNA-based immunization by in vivo transfection of dendritic cells. Nat. Med. 10:1122-1128.

29. Corr, M., Lee, D.J., Carson, D.A., and Tighe, H. 1996. Gene vaccination with naked plasmid DNA: mechanism of CTL priming. J. Exp. Med. 184: $1555-1560$

30. Doe, B., Selby, M., Barnett, S., Baenzinger, J., and Walker, C.M. 1996. Induction of cytotoxic T lymphocytes by intramuscular immunization with plasmid DNA is facilitated by bone marrow-derived cells. Proc. Natl. Acad. Sci. USA. 93:8578-8583.

31. Bevilacqua, M.P. 1993. Endothelial-leukocyte adhesion molecules. Annu. Rev. Immunol. 11:767-804.

32. Hohlfeld, R., and Engel, A.G. 1994. The immunobiology of muscle. Immunol. Today. 15:269-274 